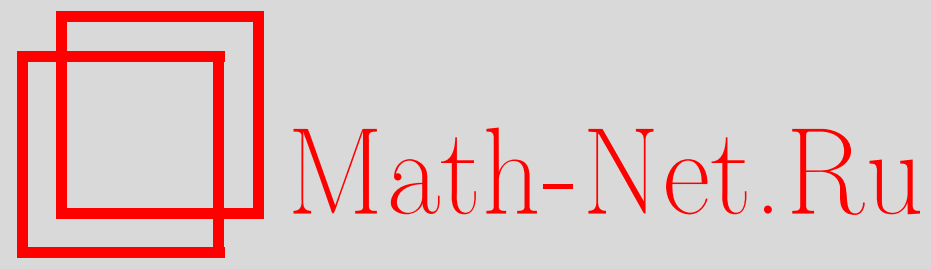

М. М. Арсланов, Теоретико-модельные свойства тьюринговых степеней разностной иерархии Ершова, Совр. пробл. матем., 2011, выпуск 15, 5-14

DOI: https://doi.org/10.4213/spm26

Использование Общероссийского математического портала Math-Net.Ru подразумевает, что вы прочитали и согласны с пользовательским соглашением http://www . mathnet.ru/rus/agreement

Параметры загрузки:

IP : 3.85 .73 .92

26 апреля 2023 г., 02:45:21

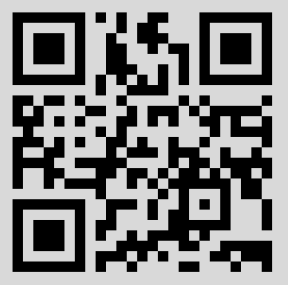




\title{
Теоретико-модельные свойства тьюринговых степеней разностной иерархии Ершова
}

\author{
М. М. Арсланов \\ Казанский (Приволжский) федералъный университет
}

В настоящее время накоплено довольно много результатов, относящихся к разработке структурной теории (тьюринговых) степеней неразрешимости, содержащих множества из различных уровней иерархии Ершова. Следующим естественным шагом должно было быть систематическое рассмотрение теоретико-модельных свойств этих структур, но в этой области сделано пока еще очень немного.

Эти исследования берут начало со следующего характеристического свойства множеств по тьюринговой сводимости (для краткости Т-сводимости) расположенных ниже $\varnothing^{\prime}-$ степени неразрешимости проблемы остановки машины Тьюринга. Множество $A$ Т-сводится к множеству $\varnothing^{\prime}$ (для краткости $A \leqslant$ т $\varnothing^{\prime}$ ) тогда и только тогда, когда существует такая вычислимая функция $f(s, x)$, что $A(x)=\lim _{s} f(s, x)$. Здесь $A(x)$ - характеристическая функция множества $A$ :

$$
A(x)= \begin{cases}1, & \text { если } x \in A, \\ 0, & \text { если } x \notin A .\end{cases}
$$

Таким образом, условие $A \leqslant_{\text {т }} \varnothing^{\prime}$ эквивалентно тому, что множество $A$ может быть вычислимо аппроксимируемо в следующем смысле: существует такое множество равномерно вычислимых последовательностей $\{f(0, x), f(1, x), \ldots, f(s, x), \ldots \mid x \in \omega\}$, состоящих из 0 и 1 , что для каждого $x$ предел последовательности $f(0, x), f(1, x), \ldots$ существует и равен значению $A(x)$.

Ершов [1]-[3] построил иерархию множеств, расположенных ниже $\varnothing^{\prime}$, которая теперь известна как иерархия Ершова. Место множества $A$ в иерархии определяется по количеству изменений в аппроксимиции множества $A$ с помощью описанной выше вычислимой последовательности, т.е. по количеству различных пар соседних элементов этой последовательности. Таким образом, иерархия Ершова ранжирует степени множеств, входящих в иерархию, в соответствии с алгоритмической сложностью распознавания их элементов. Иерархия Ершова состоит из конечных и бесконечных уровней. $\mathrm{K}$ конечным уровням иерархии относятся множества, у которых количество таких изменений ограничено некоторым натуральным числом. В противном случае множество принадлежит одному из бесконечных уровней иерархии Ершова. Таким образом, конечный уровень $n, n \geqslant 1$, иерархии Ершова составляют $n$-в.п. множества, которые в каноническом виде выглядят как

$$
A=\bigcup_{i=0}^{[(n-1) / 2]}\left\{\left(R_{2 i+1}-R_{2 i}\right) \cup\left(R_{2 i}-R_{2 i+1}\right)\right\},
$$

где $R_{0}, R_{1}, R_{2}, \ldots, R_{n-1}$ - такие в.п. множества, что $R_{0} \subseteq R_{1} \subseteq R_{2} \subseteq \cdots \subseteq R_{n-1}$. (Если $n$ - число нечетное, то $R_{n}=\varnothing$.) Бесконечные уровни иерархии определяются с помощью бесконечных конструктивных ординалов. (Конечные уровни иерархии Ершова впервые под другими названиями были определены и изучены в работах Голда [4], Путнама [5] и др. Аддисон [6] рассмотрел общий метод построения "разностных" иерархий. В частности, построенная им иерархия, порожденная в.п. множествами, приводит к тем же классам $n$-в.п. множеств. В работе Аддисона получены также некоторые свойства $n$-в.п. множеств, например доказана теорема об иерархии.) 
Как оказалось, возникающая иерархия множеств исчерпывает всю совокупность множеств, расположенных по Т-сводимости ниже $\varnothing^{\prime}$. Каждый следующий уровень иерархии содержит все предыдущие, но не совпадает ни с одним из них, при этом уровни иерархии устроены настолько "равномерно", что высказывалась даже гипотеза (Доуни [7]), что полурешетки тьюринговых степеней множеств конечных уровней иерархии начиная со второго неразличимы на языке первого порядка. Это предположение получило известность как гипотеза Доуни и вызвало целый ряд публикаций.

Степень а называется $n$-в.n. степенъю, если она содержит некоторое $n$-в.п. множество. Если она содержит $n$-в.п. множества и не содержит $(n-1)$-в.п. множества, то называется собственной n-в.n. степенъю. Множество всех $n$-в.п. степеней мы обозначим через $\mathscr{D}_{n}$ (как обычно, $\mathscr{R}=\mathscr{D}_{1}$ обозначает совокупность всех в.п. степеней). Через $\mathscr{D}\left(\leqslant \mathbf{0}^{\prime}\right)$ обозначается совокупность степеней, по тьюринговой сводимости расположенных ниже $\mathbf{0}^{\prime}$, а множество всех тьюринговых степеней - через $\mathscr{D}$. Все эти совокупности образуют верхние полурешетки относительно Т-сводимости: наименьшей верхней гранью степеней $\mathbf{a}$ и $\mathbf{b}$, содержащих соответственно множества $A$ и $B$, является степень множества

$$
A \oplus B=\{2 x \mid x \in A\} \cup\{2 x+1 \mid x \in B\} .
$$

Мы придерживаемся обозначений, принятых в книге Соара [8]. В частности, частично вычислимые функционалы (с оракулом или без оракула) обозначаются заглавными греческими буквами, нумерация всех частично вычислимых функционалов (с оракулом или без оракула) обозначается через $\left\{\Phi_{e}\right\}_{e \in \omega} ;\left\{W_{e}\right\}_{e \in \omega}$ означает стандартную нумерацию всех в.п. множеств.

Первые результаты в исследовании структурной теории $n$-в.п. степеней были получены в 70-х годах прошлого столетия, когда Купер [9] установил существование степени, содержащей $d$-в.п. множество, но не содержащей в.п. множества (т.е. существование собственной $d$-в.п. степени), а Лахлан (не опубликовано) доказал, что для любого $n>1$ под каждой собственной $n$-в.п. степенью находится некоторая невычислимая в.п. степень. Эти два результата показывают, что совокупности $n$-в.п. степеней отличаются как от совокупности в.п. степеней, так и от совокупности всех степеней, расположенных ниже $\mathbf{0}^{\prime}$ (так как по известной теореме Сакса существуют минимальные степени $<\mathbf{0}^{\prime}$, а по упомянутой теореме Лахлана никакая $n$-в.п. степень не может быть минимальной).

Эти работы вызвали определенный интерес. Обобщая теорему Купера, Лерман и Хей (не опубликовано, см. [10]) установили, что для любого $n>1$ существуют $(n+1)$-в.п. степени с и $\mathbf{d}$ такие, что интервал $\{\mathbf{b} \mid \mathbf{c} \leqslant \mathbf{b} \leqslant \mathbf{d}\}$ не содержит $n$-в.п. степеней. Они также отметили, что сочетание предложенного Купером для доказательства существования собственной $d$-в.п. степени метода с методом разрешения позволяет построить под каждой невычислимой в.п. степени собственную $d$-в.п. степень. Позднее автор [11], сочетая метод Купера с методом кодирования Сакса, построил собственную $d$-в.п. степень и над каждой не Т-полной в.п. степенью.

Более активные исследования по разработке структурной теории $n$-в.п. (в основном $d$-в.п.) степеней начались после публикаций автора [11], [12] и Доуни [7], в которых установлено, что элементарные теории в.п. степеней и $n$-в.п. степеней различны. Автором доказано, что для любого $n \geqslant 1$ и для каждой $n$-в.п. степени $\mathbf{a}>\mathbf{0}$ существует $d$-в.п. степень $\mathbf{d}<\mathbf{0}^{\prime}$ такая, что $\mathbf{a} \cup \mathbf{d}=\mathbf{0}^{\prime}$. Ранее в работах Ейтса и Купера (не опубликовано, см. статью Миллера [13]) было установлено, что в полурешетке в.п. степеней такой результат не имеет места. Отсюда следует, что эти теории различны на $\Sigma_{3}^{0}$-уровне. Доуни доказал возможность вложения четырехэлементной решетки $\diamond$, называемой также ромбом, в $d$-в.п. степени с сохранением $\mathbf{0}$ и $\mathbf{0}^{\prime}$ (ранее Лахланом [14] было установлено, что в полурешетке в.п. степеней такой результат также не имеет места), а Купер, Лахлан, Лемпп, Соар и Харрингтон [15] установили, что упорядочение $n$-в.п. степеней неплотно для любого $n>1$. Таким образом, эти теории различны уже на $\Sigma_{2}^{0}$-уровне (на $\Sigma_{1}^{0}$-уровне теории $n$-в.п. степеней при всех $n \geqslant 1$ совпадают, что 
следует из приведенного выше результата Лахлана и теорем Мучника [16] и Сакса [17] о возможности вложения в $\mathscr{R}$ произвольного счетного частично упорядоченного множества, что означает также разрешимость их $\Sigma_{1}^{0}$-теорий). В работе [7] Доуни сформулировал также свою ставшую знаменитой гипотезу об элементарной эквивалентности упорядочений $n$ - и $m$-в.п. степеней при $n \neq m, n, m>1$.

В настоящее время структурная теория $n$-в.п. степеней достаточно хорошо разработана, что нельзя сказать об изучении теоретико-модельных свойств этих структур. К крупным достижениям, полученным в этом направлении за последние годы, относятся кроме упомянутых работ автора и Доуни следующие:

- полурешетки $d$-в.п. и 3-в.п. степеней не элементарно эквивалентны (опровержение гипотезы Доуни; автор, Калимуллин, Лемпп [18]; в этой работе приведен также первый пример определимой в полурешетке $\mathscr{D}_{n}$ бесконечной совокупности в.п. степеней);

- в.п. степени в сигнатуре $\{\leqslant\}$ не образуют $\Sigma_{1}$-подструктуру $n$-в.п. степеней ни для какого $n \geqslant 2$ (Янг и Ю [19]);

- для любого $m \geqslant 1$ частично упорядоченные множества $m$-низких в.п. и $m$-низких $d$-в.п. степеней не элементарно эквивалентны (Ямалеев [20]; этот результат при $m=1$ независимо получен М. Файзрахмановым).

Несмотря на многочисленные усилия, ответить на целый ряд естественно возникающих вопросов пока не удается.

Не разрешена проблема определимости (с параметрами или без параметров) в.п. степеней в структурах $n$-в.п. степеней при $n>1$ (в более общей постановке - проблема определимости $m$-в.п. степеней в структурах $n$-в.п. степеней при $1 \leqslant m<n)$.

Не исследована проблема определимости (с параметрами или без параметров) в $\Delta_{2}^{0}$-степенях множества всех $n$-в.п. степеней для некоторого (всех) $n>1$. (Представляет интерес нахождение естественных множеств $n$-в.п. степеней, определимых с параметрами в $\Delta_{2}^{0}$-степенях.) Не известны также ответы на следующие вопросы. Существуют ли определимые в $\mathscr{D}_{n}$, $n \geqslant 1$, индивидуальные $n$-в.п. степени, отличные от $\mathbf{0}$ и $\mathbf{0}^{\prime}$ ? Если $\mathscr{D}_{n}, n \geqslant 1$, фиксировано, то существуют ли множества $m$-в.п. степеней $(1 \leqslant m \leqslant n) C$ и $n$-в.п. степени $\mathbf{a}, \mathbf{b}$ такие, что $|C \cap[\mathbf{a}, \mathbf{b}]|=1 ?$

Проблемы определимости в степенных структурах $\mathscr{D}_{n}$ мы рассмотрим подробнее далее.

Остается открытой проблема элементарной эквивалентности структур $n$-в.п. степеней при различных $n>2$. В недавней работе автора, Калимуллина и Лемппа [18] установлено, что полурешетки $\mathscr{D}_{2}$ и $\mathscr{D}_{3}$ различны, т.е. следующее $\Sigma_{2}^{0}$-предложение $\varphi$ истинно в $\mathscr{D}_{2}$ и ложно B $\mathscr{D}_{3}$ :

$$
\varphi \doteq(\exists \mathbf{d}>\mathbf{0})(\exists \mathbf{e}>\mathbf{d})(\exists \mathbf{f}>\mathbf{e})\{(\forall \mathbf{u} \leqslant \mathbf{f})[\mathbf{u} \leqslant \mathbf{e} \vee \mathbf{e} \leqslant \mathbf{u} \cup \mathbf{d}] \&(\forall \mathbf{u} \leqslant \mathbf{e})[\mathbf{u} \leqslant \mathbf{d} \vee \mathbf{d} \leqslant \mathbf{u}]\} .
$$

Этот результат опровергает предположение Доуни [7] о попарной элементарной эквивалентности этих структур. Однако проблема элементарной эквивалентности полурешеток $\mathscr{D}_{n}$ и $\mathscr{D}_{m}$ при $m \neq n$ и $n, m \geqslant 3$ все еще остается открытой. Мы предполагаем, что теории этих полурешеток попарно различны и отличающее их предложение может быть получено подходящим обобщением приведенного выше предложения $\varphi$ на соответствующие уровни иерархии. Но доказательство этого утверждения, если идти по пути простого обобщения приведенного в [18] доказательства, может оказаться технически весьма сложным.

Если полурешетки $\mathscr{D}_{n}$ и $\mathscr{D}_{n+1}$ не элементарно эквивалентны для некоторого $n \geqslant 1$, то возникает вопрос: не является ли $\mathscr{D}_{n} \Sigma_{k}$-подструктурой $\mathscr{D}_{n+1}$ для некоторого $k \geqslant 1$ ? По определению структура $\mathscr{L}$ является $\Sigma_{k}$-подструктурой структуры $\mathscr{P}$, если $\mathscr{L} \subseteq \mathscr{P}$ и для любой $\Sigma_{k}$-формулы $\varphi\left(x_{1}, \ldots, x_{r}\right)$ и для любых $a_{1}, \ldots, a_{r} \in \mathscr{L}$

$$
\mathscr{L} \models \varphi\left(a_{1}, \ldots, a_{r}\right) \Longleftrightarrow \mathscr{P} \models \varphi\left(a_{1}, \ldots, a_{r}\right) .
$$

В этом случае пишем $\mathscr{L} \preceq \Sigma_{k} \mathscr{P}$. 
Известно (Янг и Ю [19]), что в.п. степени в сигнатуре $\{\leqslant\}$ не образуют $\Sigma_{1}$-подструктуру $n$-в.п. степеней ни для какого $n \geqslant 2$. Арсланов и Ямалеев недавно доказали, что существуют такие в.п. степень $\mathbf{d}$ и $d$-в.п. степень $\mathbf{e}$, что $\mathbf{0}<\mathbf{d}<\mathbf{e}$ и для любой $d$-в.п. степени $\mathbf{c} \leqslant \mathbf{e}$, либо $\mathbf{c} \leqslant \mathbf{d}$, либо $\mathbf{d} \leqslant \mathbf{c}$, но существует такая 3-в.п. степень $\mathbf{u} \leqslant \mathbf{e}$, что $\mathbf{u}$ несравнима с $\mathbf{d}$. Отсюда следует, что $\Sigma_{1}$-предложение

$$
\exists u(\mathbf{0}<\mathbf{d}<\mathbf{e} \& u<\mathbf{e} \& u \nless \mathbf{d} \& \mathbf{d} \nless u)
$$

истинно в $\mathscr{D}_{3}$ и ложно в $\mathscr{D}_{2}$, поэтому $\mathscr{D}_{2} \npreceq \Sigma_{1} \mathscr{D}_{3}$. Недавно стало известно, что Шор и Сламан (частное сообщение, не опубликовано) доказали, что $\mathscr{D}_{m} \npreceq \Sigma_{1} \mathscr{D}_{n}$ при всех $1 \leqslant m<n$.

Одной из наиболее трудных открытых проблем является проблема разрешимости $\exists \forall$-теории $n$-в.п. степеней для каждого $n \geqslant 1$. Как заметил Шор [21] (для случая в.п. степеней), по произвольной конечной решетке $\mathscr{L}$ легко построить $\Sigma_{2}^{0}$-предложение, которое истинно в $\mathscr{D}_{n}$ тогда и только тогда, когда $\mathscr{L}$ вложима в $\mathscr{D}_{n}$. Например, вложимость ромба в структуру $d$-в.п. степеней эквивалентна истинности следующего $\Sigma_{2}^{0}$-предложения:

$$
\exists a, b, c, d\{(a<b, c<d) \& \forall x(x \leqslant b, c \rightarrow x \leqslant a) \& x \geqslant b, c \rightarrow x \geqslant d)\} .
$$

Однако проблема описания конечных решеток, вложимых в $\mathscr{D}_{n}, n \geqslant 1$, вероятно является еще более трудной задачей. Существует множество публикаций, посвященных этой проблеме (библиографию работ см., например, в статье Лермана [22]), но окончательный результат вряд ли стоит ожидать в ближайшем будущем.

Известно также (Лерман [23]), что проверка истинности в $\mathscr{D}_{n}, n \geqslant 1$, произвольного $\Sigma_{2}^{0}$-предложения эквивалентна проблеме распознавания возможности по произвольному набору пар конечных решеток $\left\{\mathscr{P}, \mathscr{Q}_{i}\right\}, 1 \leqslant i \leqslant m$, где $\mathscr{Q}_{i}-$ расширение $\mathscr{P}$ как частично упорядоченного множества, и определить по данному вложению $\mathscr{P}$ в $\mathscr{D}_{n}$, существует ли вложение некоторого $\mathscr{Q}_{i}$ в $\mathscr{D}_{n}$, расширяющее вложение $\mathscr{P}$. В некоторых частных случаях эта проблема решается положительно (например, Сламан и Соар [24], [25] для случая $m=1$ ), но до получения общего решения еще далеко. Приведем пример, который иллюстрирует трудность проблемы для $\mathscr{D}_{2}$ даже при $m=1$.

Пусть

$$
\mathscr{P}=\{a, b, c \mid a<b<c\}, \quad \mathscr{Q}=\{a, b, c, d \mid a<b<c, a<d<c, b \nless d, d \nless b\} .
$$

Ясно, что $\mathscr{Q}$ расширяет $\mathscr{P}$, и пусть $f: \mathscr{P} \mapsto \mathscr{D}_{2}-$ такое вложение $\mathscr{P}$ в $\mathscr{D}_{2}$, что $f(a)=\mathbf{0}$. Следующая теорема автора, Калимуллина и Лемппа [18] устанавливает, что при этом может не существовать расширяющего $\mathscr{P}$ вложения $\mathscr{Q}$ в $\mathscr{D}_{2}$.

Теорема 1. Существуют такие в.п. степень $\mathbf{b}>\mathbf{0} u d$-в.n. степень $\mathbf{c}>\mathbf{b}$, что для любой в.п. степени $\mathbf{d}$, если $\mathbf{d} \leqslant \mathbf{c}$, то либо $\mathbf{b} \leqslant \mathbf{d}, л и б о ~ \mathbf{d} \leqslant \mathbf{b}$.

Некоторые теоретико-модельные свойства полурешеток $\mathscr{D}_{n}, n \geqslant 1$, так же, как и решеток n-в.п. множеств $\mathscr{C}_{n}, n \geqslant 1$ ( $\mathscr{C}_{1}=\mathscr{C}$ обозначает решетку всех в.п. множеств), можно вывести из соответствующих результатов для полурешеток $\mathscr{R}$ и $\mathscr{C}$, которые значительно более тщательно изучены. Например, так же, как и в вычислимо перечислимом случае, следующий результат может быть получен с помощью теорем Лахлана [26] о гипергиперпростых множествах и булевых алгебрах.

Теорема 2. Для любого $n \geqslant 1$ решетка $\mathscr{C}_{n}$ всех $n$-в.n. множеств не имеет вычислимого представления, т.е. она не изоморфна никакому вычислимому частичному порядку.

ДокАзАтЕЛьство. При $n=1$ результат непосредственно следует из теоремы Лахлана [26] о том, что любая $\Sigma_{3}^{0}$-булева алгебра изоморфна булевой алгебре в.п. надмножеств некоторого гипергиперпростого множества, и теоремы Фейнера [27] о существовании $\Sigma_{3}^{0}$-булевой алгебры, которая не имеет вычислимого представления. 
Теперь пусть $n \geqslant 2$ и $A$ - произвольное гипергиперпростое множество. Если $A \subseteq B, B$ является $n$-в.п. множеством и разность $B-A$ бесконечна, то она ко-в.п. (т.е. имеет в.п. дополнение). Действительно, $B-A$ должна быть $2 k$-в.п. множеством для некоторого четного $2 k$ (в противном случае $\omega-A$ не иммунна) и

$$
B-A=\left(A_{1}-A_{2}\right) \cup\left(A_{3}-A_{4}\right) \cup \cdots \cup\left(A_{2 k-1}-A_{2 k}\right)
$$

для некоторых в.п. множеств $A_{1} \supset A_{2} \supset \cdots \supset A_{2 k}$. Теорема Лахлана о гипергипериммунной разности в.п. множеств гласит, что если в.п. множества $X$ и $Y$ таковы, что $X \supset Y$ и $X-Y$ гипергипериммунна, то существует вычислимое множество $R$ такое, что $X-Y \subseteq R \subseteq X$. Отсюда следует, что существуют такие вычислимые множества $R_{1}, R_{2}, \ldots, R_{k}$, что

$$
A_{2 i-1}-A_{2 i} \subseteq R_{i} \subseteq A_{2 i-1}, \quad B-A=\overline{R_{1}} \cup\left\{\bigcup_{i=1}^{k}\left(A_{2 i} \cap\left\{\overline{\bigcup_{m>2 i} R_{m}}\right\}\right)\right\} .
$$

Это значит, что множество $A \cup\{\omega-B\}$ вычислимо перечислимо.

По теореме Лахлана множество $B$ также в.п., поэтому булевы алгебры $\mathscr{C}(A)$ и $\mathscr{C}_{n}(A)$ coответственно в.п. и $n$-в.п. надмножеств (по модулю конечных разностей) гипергиперпростого множества $A$ совпадают.

При изучении степеней изоморфных копий $\mathscr{D}_{n}$ (для краткости - степеней представлений $\mathscr{D}_{n}$ ) весьма полезным является следующий результат, принадлежащий Шору [28], доказанный для в.п. степеней и в более общей формулировке).

Теорема 3. Пусть $A$ - произвольное $\Pi_{2}^{0}$ множество. Существует такая частичная решетка (т.е. верхняя полурешетка, в которой наибольшая верхняя грань существует не для всех пар элеиентов) $\mathscr{L}$, что:

(1) $\mathscr{L}$ изоморфно вкладьвается в любую полурешетку $\mathscr{D}_{n}, n \geqslant 1$;

(2) если $\mathscr{L}$ изоморфно вкладывается в какую-нибудь полурешетку $\mathscr{S}$, то множество $A$ вычислимо относительно (тъюрингова) скачка любого представления $\mathscr{S}$.

В частности, $A$ вычислимо относительно скачка любого представления каждой полурешетки $\mathscr{D}_{n}, n \geqslant 1$. Таким образом, справедливо

СлеДСТВИЕ (Шор [28]). Ни одна из полурешеток $\mathscr{D}_{n}, n \geqslant 1$, не имеет вычислимого представления. Более того, степень любого представления кажсой из них больше или равна $\mathbf{0}^{\prime}$.

Доказательство следующей теоремы может быть получено фактически повторением доказательства Шора предыдущего результата. (Переход от степени $\mathbf{0}^{\prime}$ к высокой степени $\mathbf{h}$ осуществляется с помощью техники high permitting.)

ТЕорема 4. Для любой высокой в.n. степени $\mathbf{h}$ полурешетка $\mathscr{D}_{n}(\leqslant \mathbf{h})$ не имеет вычислимого представления.

По-видимому, справедливо и более общее утверждение: для любой в.n. cmenени а > 0 nолурешетка $\mathscr{D}_{n}(\leqslant \mathbf{a})$ не имеет вычислимого представления. Спектры степеней представлений как полурешеток $\mathscr{D}_{n}, n \geqslant 1$, так и их фрагментов $\mathscr{D}_{n}(\leqslant \mathbf{a})$ совершенно не исследованы.

Следующая теорема содержится в работе Лермана, Шора и Соара [29] для случая $n=1$, но приведенное там доказательство проходит и в общем случае.

Теорема 5. Для любого $n \geqslant 1$ полурешетка $\mathscr{D}_{n}$ не счетно категорична.

ДокАзАтЕЛЬство. Лерман, Шор и Соар [29] определили счетное множество попарно не изоморфных конечных частичных решеток (наибольшая нижняя грань существует не для всех пар степеней), порожденных тремя элементами, каждая из которых вкладывается в $\mathscr{R}$ и, следовательно, в $\mathscr{D}_{n}$ для каждого $n>1$. 
Каждая такая частичная решетка производит определенный 3-тип, реализуемый в $\mathscr{D}_{n}$. Теперь утверждение следует из теоремы Рыль-Нардзевского о счетной категоричности (см. Ершов и Палютин [30, с. 194]).

Рассмотрим более подробно проблемы определимости в полурешетках $n$-в.п. степеней. Одним из возможных способов доказательства определимости $m$-в.п. степеней в $n$-в.п. степенных структурах при $m<n$ является следующий:

а) находится некоторое бесконечное определимое в $\mathscr{D}_{n}$ множество $\mathscr{S} m$-в.п. степеней;

б) доказывается, что степени из $\mathscr{S}$ порождают под операциями $\cup$ и $\cap$ полурешетку $\mathscr{D}_{n}$ (там, где она определена).

В этих исследованиях активно используются возможные способы разложения $n$-в.п. степеней на две несравнимые степени, поэтому начнем изложение с рассмотрения этих вопросов.

ОПРЕДЕЛЕНИЕ. Разложением степени $\mathbf{d}$ называются такие степени $\mathbf{d}_{0}$ и $\mathbf{d}_{1}$, что $\mathbf{d}_{0}, \mathbf{d}_{1}<\mathbf{d}$ и $\mathbf{d}=\mathbf{d}_{0} \cup \mathbf{d}_{1}$. Говорят, что это разложение над степенъю $\mathbf{a}<\mathbf{d}$, если $\mathbf{a} \leqslant \mathbf{d}_{0}, \mathbf{d}_{1}$. Наконец, разложение $\mathbf{d}$ на $\mathbf{d}_{0}$ и $\mathbf{d}_{1}$ является разложением, которое минует верхний конус степеней $\mathbf{b}$, если $\mathbf{b} \nless \mathbf{d}_{i}, i \leqslant 1$.

Ниже приводятся все основные результаты о разложениях $n$-в.п. степеней при различных $n \geqslant 1$, которые на сегодняшний день нам известны.

ТЕОрема 6 (Сакс [31]). Каждая в.п. степень а >0 разложима на две в.п. степени, минуя верхний конус степеней $\mathbf{b}$, для любой $\Delta_{2}^{0}$-степени $\mathbf{b}>0$.

Теорема 7 (Лахлан [32]). Существуют такие в.n. степени $\mathbf{a} u \mathbf{b}, \mathbf{a}<\mathbf{b}$, что $\mathbf{b}$ не разложима в в.п. степенях нада.

Теорема 8 (Робинсон [33]). Каждая в.n. степень а > $\mathbf{0}$ разложима на две низкие в.п. степени над любой низкой в.п. степенъю.

Теорема 9 (Купер [34] для случая b = 0, Купер и Ли [35] для общего случая). Каждая d-в.п. степень $\mathbf{a}>\mathbf{0}$ разложима в $d$-в.n. степенях над произвольной в.п. степенъю $\mathbf{b}<\mathbf{a}$.

Теорема 10 (Купер, Лахлан, Лемпп, Соар и Харрингтон [15]). Не каждая n-в.n. cmeпень (даже в.п. степень) d разложима (даже в $\omega$-в.n. степенях) над произвольной $d$-в.n. степенью $\mathbf{a}<\mathbf{d}$, для любого $n>1$.

Теорема 11 (Арсланов, Купер и Ли [35], [36]). Любая в.n. степень а > 0 разложима в d-в.n. степенях над произвольной низкой $d$-в.n. степенъю $\mathbf{b}<\mathbf{a}$.

Теорема 12 (Ли [37]). Каждая d-в.n. степень $\mathbf{d}>\mathbf{0}$ разложима в $d$-в.n. степенях над каждой низкой d-в.n. степенъю $\mathbf{x}<\mathbf{d}$.

ТЕорема 13 (Шор и Сламан [38]). Пусть степени $\mathbf{d}, \mathbf{a}$ u b maковы, что $\mathbf{d}$ является $n$-в.n. для некоторого $n \geqslant 1, a \mathbf{a} u \mathbf{b}$ такие $\Delta_{2}^{0}$-степени, что $\mathbf{a} \ngtr \mathbf{b}$. Тогда $\mathbf{d}$ можно разложить над а в $\Delta_{2}^{0}$-степенях, минуя верхний конус степеней $\mathbf{b}$.

Ясно, что последний результат нельзя усилить, рассматривая разложения на $d$-в.п. степени (в [15] доказано, что этого нельзя сделать даже при $\mathbf{a}=\mathbf{0}$ ). Ямалеев [20] доказал, что такое разложение возможно при $\mathbf{a}=\mathbf{0}$, если $\mathbf{d}$ является собственно $d$-в.п. степенью, a $\mathbf{b}-$ такая невычислимая $\Delta_{2}^{0}$-степень, что между $\mathbf{d}$ и $\mathbf{b}$ нет в.п. степеней.

ТЕОРема 14 [20]. Пусть собственно d-в.п. степени $\mathbf{d}$ u $\mathbf{b}$ таковъ, что $\mathbf{d}>\mathbf{b}$ и интервал $(\mathbf{d}, \mathbf{b})$ не содержит в.n. степени. Тогда $\mathbf{d}$ можно разложить на две d-в.n. степени, минуя верхний конус степеней над $\mathbf{b}$.

Следовательно, каждая собственная $d$-в.п. степень b обладает следующим свойством. 
Для любой d-в.п. степени $\mathbf{d}>\mathbf{b}$ существует такая $d$-в.п. степень $\mathbf{a}$, что $\mathbf{b}<\mathbf{a} \leqslant \mathbf{d}, u \mathbf{a}$ разложима в d-в.п. степенях, минуя верхний конус степеней $\mathbf{b}$.

Действительно, если между степенями $\mathbf{d}$ и $\mathbf{b}$ существует некоторая в.п. степень $\mathbf{a}$, тогда $\mathbf{a}$ разложима, минуя верхний конус степеней над $\mathbf{b}$, по теореме Сакса о разложении; если же между $\mathbf{b}$ и $\mathbf{d}$ нет в.п. степеней, то $\mathbf{d}$ сама таким образом разложима по теореме Ямалеева.

Теперь рассмотрим формулу

$$
\varphi(x) \doteq(\exists b>x)(\forall d)\left[x<d \leqslant b \rightarrow\left(\forall d_{0}, d_{1}\right)\left[d=d_{0} \cup d_{1} \rightarrow x \leqslant d_{0} \vee x \leqslant d_{1}\right]\right] .
$$

Из вышеизложенного следует, что

$$
\mathscr{D}_{2}=\varphi(\mathbf{a}) \Rightarrow \mathbf{a} \text { в.п. }
$$

Если бы выполнялась и обратная импликация а в.п. $\Rightarrow \mathscr{D}_{2} \models \varphi(\mathbf{a})$, то определимость в.п. степеней в $\mathscr{D}_{2}$ была бы установлена. Однако не каждая в.п. степень обладает этим свойством. Действительно, если $\mathscr{D}_{2}=\varphi(\mathbf{a})$ для некоторой в.п. степени, то пусть $\mathbf{b}>\mathbf{a}-$ такая $d$-в.п. степень, которая не разложима, минуя верхний конус степеней $\mathbf{a}$. Тогда между $\mathbf{a}$ и $\mathbf{b}$ нет в.п. степеней, за исключением а; в этом случае говорят, что a изолирует b. Но в работе автора, Лемппа и Шора [39] установлено, что между любыми двумя в.п. степенями $\mathbf{a}_{0}<\mathbf{a}_{1}$ существует в.п. степень, которая не изолирует никакую $d$-в.п. степень. Для каждой такой степени а имеем $\mathscr{D}_{2} \models \neg \varphi(\mathbf{a})$.

Следующая теорема показывает, что тем не менее существует бесконечная совокупность в.п. степеней а, удовлетворяющих формуле $\varphi$.

Теорема 15 (Арсланов, Калимуллин, Лемпп [18]). Существует бесконечно много различных d-в.n. степеней $\mathbf{d}$ и в.п. степеней $\mathbf{b}<\mathbf{d}$ таких, что любая $d$-в.п. степень $\mathbf{a}, \mathbf{b}<\mathbf{a} \leqslant \mathbf{d}$, не разложима в d-в.п. степенях, минуя верхний конус степеней над $\mathbf{b}$.

Отсюда следует, что множество

$$
\mathscr{S}=\left\{\mathbf{x} \geqslant \mathbf{0} \mid(\exists \mathbf{y}>\mathbf{x})(\forall \mathbf{z})\left(x<z \leqslant y \rightarrow\left(\forall \mathbf{z}_{\mathbf{0}}, \mathbf{z}_{\mathbf{1}}\right)\left(\mathbf{z}_{\mathbf{0}} \cup \mathbf{z}_{\mathbf{1}}=\mathbf{z} \& \mathbf{z}_{\mathbf{0}} \mid \mathbf{z}_{\mathbf{1}} \rightarrow \mathbf{x} \leqslant \mathbf{z}_{\mathbf{0}} \vee \mathbf{x} \leqslant \mathbf{z}_{\mathbf{1}}\right)\right)\right\}
$$

состоит только из в.п. степеней, бесконечно и определимо в $\mathscr{D}_{2}$ формулой

$$
\left.\varphi(x)=(\exists \mathbf{y}>\mathbf{x})(\forall z)\left(x<z \leqslant y \rightarrow\left(\forall \mathbf{z}_{\mathbf{0}}, \mathbf{z}_{1}\right)\left(\mathbf{z}_{\mathbf{0}} \cup \mathbf{z}_{\mathbf{1}}=\mathbf{z} \& \mathbf{z}_{\mathbf{0}} \mid \mathbf{z}_{\mathbf{1}} \rightarrow \mathbf{x} \leqslant \mathbf{z}_{\mathbf{0}} \vee \mathbf{x} \leqslant \mathbf{z}_{1}\right)\right)\right\} .
$$

Теперь предположим, что $\mathbf{d}$ - некоторая собственная $d$-в.п. степень. Для любого разложения $\mathbf{d}$ на две $d$-в.п. степени $\mathbf{d}_{0}$ и $\mathbf{d}_{1}$ по крайней мере одна из степеней $\mathbf{d}_{i}, i \leqslant 1$, должна обладать следующим свойством.

Любая d-в.п. степень $\mathbf{u}, \mathbf{d}_{i} \leqslant \mathbf{u} \leqslant \mathbf{d}$, разложима на d-в.п. степени, минуя верхний конус cmепеней над $\mathbf{d}_{i}$.

В противном случае по теореме Ямалеева для каждой степени $\mathbf{d}_{i}, i \leqslant 1$, между $\mathbf{d}_{i}$ и $\mathbf{d}$ существует в.п. степень, и, следовательно, степень $\mathbf{d}$ сама вычислимо перечислима (как наибольшая нижняя грань таких степеней).

Ответы на следующие вопросы пока не известны. (Из вышеизложенного следует, что положительный ответ на любой из них означает определимость в.п. степеней в $\mathscr{D}_{2}$. )

Вопрос 1. Верно ли, что каждая в.п. степень $\mathbf{a}>\mathbf{0}$ является наименьшей верхней гранью двух несравнимых степеней из $\mathscr{S}$ ?

С этим вопросом связан также следующий: верно ли, что степени из $\mathscr{S}$ плотны среди в.п. степеней, т.е. верно ли, что между любыми двумя в.п. степенями $\mathbf{a}<\mathbf{b}$ найдется некоторая степень из $\mathscr{S}$ ? Положительный ответ на этот вопрос немедленно влечет положительный ответ на вопрос 1: для этого разлагаем в.п. степень $\mathbf{a}>\mathbf{0}$ на две несравнимые в.п. степени $\mathbf{a}_{0}$ и $\mathbf{a}_{1}$ (по теореме Сакса о разложении это можно сделать) и между степенями а и $\mathbf{a}_{i}, i \leqslant 1$, находим степени $\mathbf{b}_{i} \in \mathscr{S}$. Имеем $\mathbf{a}=\mathbf{b}_{0} \cup \mathbf{b}_{1}$. 
Вопрос 2. Верно ли, что для каждой в.п. степени а существует такое разложение на ( $d$-в.п. степени) $\mathbf{a}_{0}, \mathbf{a}_{1}$, что некоторые $d$-в.п. степени $\mathbf{b}_{0}, \mathbf{b}_{1}$ такие, что $\mathbf{a}_{i}<\mathbf{b}_{i}<\mathbf{a}$, не разложимы, минуя верхний конус степеней $\mathbf{a}_{i}$, для каждого $i \in\{0,1\}$ ?

Исследование проблемы определимости в.п. степеней в полурешетках $\mathscr{D}_{n}, n>2$, также можно проводить по следующей схеме.

1) Пусть $1 \leqslant m<n$ и собственные $n$-в.п. степени $\mathbf{d}$ и $\mathbf{b}$ таковы, что $\mathbf{d}>\mathbf{b}$ и интервал $(\mathbf{d}, \mathbf{b})$ не содержит $m$-в.п. степеней. Верно ли, что $\mathbf{d}$ разложима в $n$-в.п. степенях, минуя верхний конус степеней над $\mathbf{b}$ ?

2) Каждая $n$-в.п. степень а разложима на две $(n+1)$-в.п. степени $\mathbf{a}_{0}, \mathbf{a}_{1}$, так что существуют такие $(n+1)$-в.п. степени $\mathbf{b}_{0}, \mathbf{b}_{1}, \mathbf{a}_{i}<\mathbf{b}_{i}<\mathbf{a}$, не разложимые в $(n+1)$-в.п. степени, минуя верхний конус степеней над $\mathbf{a}_{i}$, для каждого $i \in\{0,1\}$.

Мы предполагаем, что для каждого $n \geqslant 1$ множества

$$
\mathscr{S}_{n}=\left\{\mathbf{x} \geqslant \mathbf{0} \mid(\exists \mathbf{y}>\mathbf{x})(\forall z)\left(x<z \leqslant y \rightarrow\left(\forall \mathbf{z}_{\mathbf{0}}, \mathbf{z}_{\mathbf{1}}\right)\left(\mathbf{z}_{\mathbf{0}} \cup \mathbf{z}_{\mathbf{1}}=\mathbf{z} \& \mathbf{z}_{\mathbf{0}} \mid \mathbf{z}_{\mathbf{1}} \rightarrow \mathbf{x} \leqslant \mathbf{z}_{\mathbf{0}} \vee \mathbf{x} \leqslant \mathbf{z}_{\mathbf{1}}\right)\right)\right\}
$$

$n$-в.п. степеней определимы в $\mathscr{D}_{n+1}$.

Мы также предполагаем, что это множество $n$-в.п. степеней порождает весь класс $n$-в.п. степеней и, таким образом, справедливо следующее свойство.

Для любого $n>1$ n-в.n. степени равномерно определимы в $\mathscr{D}_{n+1}$.

Перейдем к рассмотрению вопросов определимости с параметрами.

ТЕОРема 16. Существует бесконечная совокупность в.п. степеней, каждая из которых определима в $\mathscr{D}_{2}$ с одним параметром из $\mathscr{D}_{2}-\mathscr{R}$.

ДоКАЗАТЕЛЬСтво. Для любого $\mathbf{a} \in \mathscr{S}$ пусть $\mathbf{d}$ является такой степенью, что для любой степени $\mathbf{z}$ при $\mathbf{a}<\mathbf{z} \leqslant \mathbf{d}$ и $\mathbf{z}_{\mathbf{0}} \cup \mathbf{z}_{\mathbf{1}}=\mathbf{z}$ для некоторых $d$-в.п. степеней $\mathbf{z}_{\mathbf{0}}$ и $\mathbf{z}_{\mathbf{1}}$, и $\mathbf{z}_{\mathbf{0}} \mid \mathbf{z}_{\mathbf{1}}$, справедливо либо $\mathbf{a} \leqslant \mathbf{z}_{\mathbf{0}}$, либо $\mathbf{a} \leqslant \mathbf{z}_{\mathbf{1}}$. Ясно, что $\mathbf{d}$ не вычислимо перечислима и однозначно определяет в.п. степень а. В частности, $S \cap[\mathbf{0}, \mathbf{y}]=\{\mathbf{a}\}$.

Сламан и Вудин [40] доказали, что класс $\mathscr{R}$ в.п. степеней определим с параметрами в $\mathscr{D}$ $\left(\leqslant \mathbf{0}^{\prime}\right)$. Принципиальное место в их доказательство занимает следующая теорема.

ТеОрема 17 [40]. Пусть $\mathscr{A}$ - равномерно низкое множество $\Delta_{2}^{0}$-степеней, ограниченное некоторой низкой степенъю а. Тогда $\mathscr{A}$ определимо с параметрами в $\mathscr{D}\left(\leqslant \mathbf{0}^{\prime}\right)$.

Множество степеней $\mathscr{A}$ называется равномерно низкой совокупностъю, если существуют последовательность множеств $\langle X(n) \mid n \in \omega\rangle$ и $\varnothing^{\prime}$-вычислимая функция $f$ такие, что $\{\operatorname{deg}(X(n)) \mid n \in \omega\}=\mathscr{A}$ и $\Phi_{f(n)}^{\varnothing^{\prime}}=(X(n))^{\prime}$.

Из теоремы 17 определимость $\mathscr{R}$ с параметрами в $\mathscr{D}\left(\leqslant \mathbf{0}^{\prime}\right)$ выводится с помощью следующей теоремы.

Теорема 18 (Уэлш [41]). Существуют две равномерно вычислимые совокупности в.п. степеней $\mathscr{A}_{0}$ и $\mathscr{A}_{1}$ такие, что каждая из них ограничена некоторой низкой степенъю и каждая в.п. степенъ а является наименъшей верхней гранъю $\mathbf{a}_{0} \in \mathscr{A}_{0} u \mathbf{a}_{1} \in \mathscr{A}_{1}$.

Для доказательства этой теоремы достаточно креативное множество $K=\left\{\langle e, n\rangle \mid n \in W_{e}\right\}$ разложить по теореме Сакса на два низких Т-несравнимых множества $A_{0}$ и $A_{1}$ и определить $\mathscr{A}_{i}=\left\{e \mid\langle e, n\rangle \in A_{i}\right\}_{n \in \omega}, i \leqslant 1$.

По теореме 18 класс $\mathscr{R}$ в.п. степеней определим с параметрами в $\mathscr{D}\left(\leqslant \mathbf{0}^{\prime}\right)$.

В [15] была доказана следующая теорема.

Tеорема 19. Существуют d-в.n. множество $E$ и в.n. множество $D$ mакие, что $\varnothing<<_{\mathrm{T}}$ $D<_{\mathrm{T}} D \oplus E$ и для любого 2-в.п. множества $U$, если $U \leqslant_{\mathrm{T}} D \oplus E$, то либо $D \leqslant_{\mathrm{T}} U$, либо $U \leqslant \mathrm{~T} D$. 
Нетрудно проверить, что удовлетворение дополнительного требования

$$
(\forall e)(\exists \infty s)\left(\Phi_{e}^{D}(e)[s] \downarrow\right) \rightarrow \Phi_{e}^{D}(e) \downarrow,
$$

которое позволяет в этой теореме сделать степень множества $D$ низкой, легко сочетается с процессом удовлетворения всех остальных ее требований. Таким образом, существуют в.п. множества низкой степени, удовлетворяющие условиям приведенной теоремы. Нам не известно, существуют ли множества не низкой степени с этими свойствами, т.е. существуют ли такие $d$-в.п. степени $\mathbf{d}>\mathbf{0}$, у которых разложения на $d$-в.п. степени не могут быть низкими:

$$
(\mathbf{d}=\mathbf{a} \cup \mathbf{b}) \rightarrow \mathbf{a}^{\prime}>\mathbf{0}^{\prime} \& \mathbf{b}^{\prime}>\mathbf{0}^{\prime}
$$

Если бы приведенную выше теорему удалось усилить таким образом, то это бы означало, что описанный выше метод Сламана и Вудина принципиально не подходит для доказательства определимости $n$-в.п. степеней с параметрами в $\mathscr{D}\left(\leqslant \mathbf{0}^{\prime}\right)$. А именно, $n$-в.п. степени при $n>1$ в общем случае нельзя разложить на две низкие $n$-в.п. степени, в отличие от случая в.п. степеней.

\section{Список литературы}

[1] Ю. Л. Ершов, “Об одной иерархии множеств, I”, Алгебра и логика, 7:1 (1968), 47-74.

[2] Ю. Л. Ершов, “Об одной иерархии множеств, II”, Алгебра и логика, 7:4 (1968), 15-47.

[3] Ю. Л. Ершов, “Об одной иерархии множеств, III”, Алгебра и логика, 9:1 (1970), 34-51.

[4] E. M. Gold, "Limiting recursion", J. Symbolic Logic, 30:1 (1965), 28-48.

[5] H. Putnam, "Trial and error predicates and the solution to a problem of Mostowski", J. Symbolic Logic, 30:1 (1965), 49-57.

[6] J. W. Addison, "The method of alternating chains", Theory of Models, North Holland, Amsterdam, 1965, 1-16.

[7] R. Downey, "D.r.e. degrees and the nondiamond theorem", Bull. London Math. Soc., 21:1 (1989), $43-50$.

[8] R. I. Soare, Recursively Enumerable Sets and Degrees. A Study of Computable Functions and Computably Generated Sets, Perspect. Math. Logic, Springer-Verlag, Berlin, 1987; Р. И. Сoap, Buчислимо перечислимые множества и степени, Казан. матем. общ-во, Казань, 2001.

[9] S. B. Cooper, Degrees of Unsolvability, Ph.D. Thesis, Univ. of Leicester, 1971.

[10] R. L. Epstein, R. Haas, R. L. Kramer, "Hierarchies of sets and degrees below 0", Logic Year 1979-80, Lecture Notes in Math., 859, Springer-Verlag, Berlin, 1981, 32-48.

[11] М. М. Арсланов, “О структуре степеней ниже 0’”, Изв. вузов. Матем., 1988, № 7, 27-33.

[12] М. М. Арсланов, "Структурные свойства степеней ниже 0'”, Докл. АН СССР, 283:2 (1985), 270-273.

[13] D. P. Miller, "High recursively enumerable degrees and the anti-cupping property", Logic Year 1979-80, Lecture Notes in Math., 859, Springer-Verlag, Berlin, 1981, 230-245.

[14] A.H. Lachlan, "Lower bounds for pairs of recursively enumerable degrees", Proc. London Math. Soc. (3), 16:1 (1966), 537-569.

[15] S. B. Cooper, L. Harrington, A. H. Lachlan, S. Lempp, R. I. Soare, "The d.r.e. degrees are not dense", Ann. Pure Appl. Logic, 55:2 (1991), 125-151.

[16] А. А. Мучник, "Решение проблемы сводимости Поста и некоторых других проблем теории алгоритмов, I", Тр. ММО, 7, Изд-во Моск. ун-та, М., 1958, 391-405.

[17] G. E. Sacks, Degrees of Unsolvability, Ann. of Math. Stud., 55, Princeton Univ. Press, Princeton, NJ, 1963.

[18] M. M. Arslanov, I. Sh. Kalimullin, S. Lempp, "On Downey's conjecture”, J. Symbolic Logic, 75:2 (2010), 401-441. 
[19] Y. Yang, L. Yu, "On $\Sigma_{1}$-structural differences among Ershov hierarchies", J. Symbolic Logic, 71:4 (2006), 1223-1236.

[20] М. М. Ямалеев, "Разложимость 2-вычислимо перечислимых степеней с избеганием конусов", Изв. вузов. Матем., 2009, №6, 76-80.

[21] R. A. Shore, "The recursively enumerable degrees", Handbook of Computability Theory, Stud. Logic Found. Math., 140, North-Holland, Amsterdam, 1999, 169-197.

[22] M. Lerman, "Embeddings into the recursively enumerable degrees", Computability, Enumerability, Unsolvability, London Math. Soc. Lecture Note Ser., 224, Cambridge Univ. Press, Cambridge, 1996, 185-204.

[23] M. Lerman, The Degrees of Unsolvability. Local and Global Theory, Perspect. Math. Logic, Springer-Verlag, Berlin, 1983.

[24] T. A. Slaman, R. I. Soare, "Algebraic aspects of the computably enumerable degrees", Proc. Nat. Acad. Sci. U.S.A., 92:2 (1995), 617-621.

[25] T. A. Slaman, R. I. Soare, "Extension of embeddings in the computably enumerable degrees", Ann. of Math. (2), 154:1 (2001), 1-43.

[26] A.H. Lachlan, "The elementary theory of recursively enumerable sets", Duke Math. J., 35:1 (1968), $123-146$.

[27] L. Feiner, "Hierarchies of Boolean algebras", J. Symbolic Logic, 35:3 (1970), 365-374.

[28] R. A. Shore, "Finitely generated codings and the degrees r.e. in a degree d", Proc. Amer. Math. Soc., 84:2 (1982), 256-263.

[29] M. Lerman, R. A. Shore, R. I. Soare, "The elementary theory of the recursively enumerable degrees is not $\aleph_{0}$-categorical", Adv. in Math., 53:3 (1984), 301-320.

[30] Ю. Л. Ершов, Е. А. Палютин, Математическая логика, Наука, М., 1987.

[31] A. H. Lachlan, "A recursively enumerable degree which will not split over all lesser ones", Ann. Math. Logic, 9:4 (1976), 307-365.

[32] R.W. Robinson, "Interpolation and embedding in the recursively enumerable degrees", Ann. of Math. (2), 93:2 (1971), 285-314.

[33] S. B. Cooper, "A splitting theorem for the n-r.e. degrees", Proc. Amer. Math. Soc., 115:2 (1992), 461-471.

[34] S. B. Cooper, A. Li, "Turing definability in the Ershov hierarchy", J. London Math. Soc. (2), 66:3 (2002), 513-528.

[35] M. Arslanov, S. B. Cooper, A. Li, "There is no low maximal d.c.e. degree", MLQ Math. Log. Q., 46:3 (2000), 409-416.

[36] M. Arslanov, S. B. Cooper, A. Li, "There is no low maximal d.c.e. degree - Corrigendum", MLQ Math. Log. Q., 50:6 (2004), 628-636.

[37] A. Li, "The low splitting theorem in the difference hierarchy", New Computational Paradigms, Lecture Notes in Comput. Sci., 3526, Springer-Verlag, Berlin, 2005, 287-296.

[38] R. A. Shore, T. A. Slaman, "A splitting theorem for n-REA degrees", Proc. Amer. Math. Soc., 129:12 (2001), 3721-3728.

[39] M. M. Arslanov, S. Lempp, R. A. Shore, "On isolating r.e. and isolated d-r.e. degrees", Computability, Enumerability, Unsolvability, London Math. Soc. Lecture Note Ser., 224, Cambridge Univ. Press, Cambridge, 1996, 61-80.

[40] T. A. Slaman, W.H. Woodin, "Definability in the Turing degrees", Illinois J. Math., 30:2 (1986), 320-334.

[41] L. V. Welch, A hierarchy of families of recursively enumerable degrees and a ntheorem on bounding minimal pairs, Ph.D. Thesis, Univ. of Illinois at Urbana-Champaign, 1981. 